\title{
Decreased Infarct Volume and Intracranial Hemorrhage Associated with Intra-Arterial Nonionic Iso-Osmolar Contrast Material in an MCA Occlusion/Reperfusion Model
}

\author{
H. Morales, A. Lu, Y. Kurosawa, J.F. Clark, J. Leach, K. Weiss, and T. Tomsick
}

\begin{abstract}
BACKGROUND AND PURPOSE: Infarct volume and intracranial hemorrhage after reperfusion with nonionic low-osmolar and isoosmolar iodinated IRCM has not been previously compared. We postulated that iso-osmolar and low-osmolar iodinated contrast media exert varied effects on cerebral infarct after intra-arterial injection. We compared infarct volume and hemorrhagic changes following intra-arterial infusion of iodixanol, iopamidol, or normal saline in a rat MCA occlusion/reperfusion model.
\end{abstract}

MATERIALS AND METHODS: Infarct was induced in 30 rats by a previously validated method of MCA suture occlusion. Reperfusion was performed after 5 hours with either iodixanol $(n=9)$, iopamidol $(n=12)$, or saline $(n=9)$. MR images were obtained at both 6 and 24 hours after ischemia, followed by sacrifice. Infarct volume was measured with T2WI and DWI by semiautomatic segmentation. Incidence and area of hemorrhage were measured on brain sections postmortem.

RESULTS: T2WI mean infarct volumes were $242 \pm 89,324 \pm 70$, and $345 \pm 92 \mathrm{~mm}^{3}$ at 6 hours, and $341 \pm 147,470 \pm 91$, and $462 \pm 71 \mathrm{~mm}^{3}$ at 24 hours in the iodixanol, iopamidol, and saline groups, respectively. Differences in infarct volume among groups were significant at 6 hours $(P<.03)$ and 24 hours $(P<.05)$. In the iodixanol, iopamidol, and saline groups, mean areas for cortical intracranial hemorrhage were $0.8,18.2$, and $25.7 \mathrm{~mm}^{2}$; and 28,31 , and $56.7 \mathrm{~mm}^{2}$, respectively, for deep intracranial hemorrhage. The differences in intracranial hemorrhage area among groups were statistically significant for cortical intracranial hemorrhage $(P<.01)$.

CONCLUSIONS: Intra-arterial infusion of nonionic iso-osmolar iodixanol showed reduced infarct volume and reduced cortical intracranial hemorrhage areas in comparison with nonionic low-osmolar iopamidol and saline. Our results may be relevant in the setting of intra-arterial therapy for acute stroke in humans, warranting further investigation.

ABBREVIATIONS: IA = intra-arterial; ICH = intracranial hemorrhage; IRCM = iodinated radiographic contrast material; $H T=$ hemorrhagic transformation

U se of iodinated radiographic contrast material (IRCM) is inherent in the current diagnostic and therapeutic paradigm of stroke evaluation and therapy in humans, not only via intravenous infusion in CT angiography and CT perfusion but also via

Received January 6, 2014; accepted after revision March 2.

From the Departments of Radiology (H.M., J.L., T.T.) and Neurology (A.L., Y.K., J.F.C.), University of Cincinnati, Cincinnati, Ohio; and Department of Radiology (K.W.), University of Mississippi, Oxford, Mississippi.

This research was supported by the 2008 Neuroradiology Education and Research Fund of the American Society of Neuroradiology via the Boston Scientific Fellowship in Cerebrovascular Disease Research Award to Humberto Morales MD, Principal Investigator. It was also supported by a grant from the Society of Interventional Surgery for purchase of the custom rat head coil.

Paper previously presented at: Annual Meeting of the American Society of Neuroradiology and Neuroradiology Education and Research Foundation Symposium, May 15-20, 2010; Boston, Massachusetts.

Please address correspondence to Humberto Morales, MD, Section of Neuroradiology, Department of Radiology, University of Cincinnati Medical Center, 234 Goodman St, ML 0762, Cincinnati, OH 45219; e-mail: moralehc@ucmail.uc.edu

Evidence-Based Medicine Level 2.

http://dx.doi.org/10.3174/ajnr.A3953 intra-arterial (IA) injection of IRCM during revascularization attempts. Data identifying CTP IRCM leakage as a predictor of subsequent hemorrhagic transformation (HT) and intracerebral hemorrhage (ICH) have accumulated. ${ }^{1-3}$ Yet IRCM deposition in the brain as a sequela of IA revascularization in humans is a frequent phenomenon, commonly but not invariably associated with HT. ${ }^{4}$

Having identified relevant differences in ICH following IA infusion of nonionic low-osmolar IRCM in a rat reperfusion model, we postulated that potential clinical differences might also exist between low-osmolar and iso-osmolar IRCM with IA infusion. ${ }^{5}$ Therefore, we evaluated nonionic iso-osmolar IRCM (iodixanol, $290 \mathrm{mOsmol}$ ) versus a nonionic low-osmolar IRCM (iopamidol, $616 \mathrm{mOsmol}$ ) on brain infarct and ICH in a rodent MCA occlusion/reperfusion stroke model. To the best of our knowledge, similar experimental studies evaluating potential differences of IA IRCM on these parameters have not been previously reported. 


\section{MATERIALS AND METHODS}

The animal protocol was approved by the University Animal Care Committee and conformed to the National Institutes of Health Guide for Care and Use of Laboratory Animals (http://grants. nih.gov/grants/olaw/Guide-for-the-care-and-use-of-laboratoryanimals.pdf). Male Sprague-Dawley rats had unrestricted access to food and water and were housed with a 12-hour light-dark cycle. Throughout the study, the investigators and veterinarian staff closely monitored the rats' health status.

This was a single blinded placebo-controlled study.

\section{General Surgical Preparation}

Thirty-four male Sprague-Dawley rats (body weight $=310.7 \pm$ $12 \mathrm{~g}$ ) were anesthetized with 3\% isoflurane and maintained with $1.5 \%$ isoflurane in a mixture of $70 \% \mathrm{~N}_{2}$ and $28.5 \% \mathrm{O}_{2}$. Rectal temperature was monitored and maintained at $37 \pm 0.5^{\circ} \mathrm{C}$ with a feedback-controlled heating blanket. Four animals were excluded from the analysis due to failure of the surgical technique. They were not imaged, dying immediately following reperfusion. Subarachnoid hemorrhage was identified on postmortem analysis.

\section{Stroke Model and Drug Injection}

A previously validated method of MCA occlusion with intraluminal suture was used. ${ }^{6}$ The left common carotid artery, external carotid artery, and internal carotid artery were isolated via a ventral midline incision. A 3/0 monofilament nylon suture was used to occlude the MCA. ${ }^{7}$ The suture was inserted into the external carotid artery and advanced into the ICA until the tip occluded the junction of the MCA and anterior cerebral artery (approximately $20 \mathrm{~mm}$ beyond the carotid bifurcation until mild resistance was felt). The wound was closed temporarily, and the suture was kept in place for 5 hours. Rats were then re-anesthetized, and the ventral midline neck incision was again opened. Immediately after removal of the suture to allow reperfusion, a polyethylene- 10 tube was placed into the external carotid artery with its tip at the ICA origin The ICA was not directly catheterized. All animals initially received a 10-minute intra-arterial saline infusion of $1 \mathrm{~mL} / \mathrm{kg}$. Next, either iodixanol (290 mOsmol/kg H $\mathrm{H}_{2}$, Visipaque, $320 \mathrm{mg} \mathrm{I} / \mathrm{mL}$; GE Healthcare, Princeton, New Jersey), iopamidol (616 mOsmol/ $/ \mathrm{kg} \mathrm{H}_{2} 0$, Isovue, $300 \mathrm{mg}$ $\mathrm{I} / \mathrm{mL}$; Bracco Diagnostics, Princeton, New Jersey), or normal saline (300 mOsmol/ $/ \mathrm{kg} \mathrm{H}_{2} 0$ ) was infused $(1 \mathrm{~mL} / \mathrm{kg}$ ) for an additional 10 minutes. The external carotid artery was ligated, and the incisions were sutured. Thus, 3 groups were studied as follows: iso-osmolar iodixanol group $(n=9)$, low-osmolar iopamidol group, $(n=12)$, and saline group $(n=9)$.

\section{Monitoring of Regional Cerebral Blood Flow}

Before MCA occlusion, a small craniotomy near the vertex (5 mm lateral and $2 \mathrm{~mm}$ posterior to the bregma) was performed. This allowed cerebral blood flow estimation from the left MCA by using transcranial laser Doppler sonography perfusion measurements (PF5001; Perimed, Järfälla, Sweden) before and after MCA occlusion and after reperfusion with saline and/or IRCM infusion. ${ }^{8}$

\section{Neurologic Deficits and Mortality}

Neurologic examinations at 45 minutes and 2, 12, and 24 hours after induction of ischemia were scored according to a 7-point scale modified from Zhang et $\mathrm{al}^{9}$ : no neurologic deficit $=0$, forelimb flexion $=1$, circling to the right $=2$, impaired or lame forelimbs $=3$ and hind limbs $=4$, inability to walk $=5$, and animal death $=6$. Average scores were calculated for each group. Mortality was expressed as the number of rats dying within 24 hours before euthanasia divided by the number in each group $\times 100$.

\section{MR Imaging Protocol}

We used a custom-made single-channel coil (Resonance Innovations, Omaha, Nebraska) designed to fit a rat's head and optimized for use in a clinical 3T magnet (Signa Excite; GE Healthcare, Milwaukee, Wisconsin).

Following reperfusion at both 6 and 24 hours after ischemia, the animals were imaged in the coronal plane with the following protocol: T2WI (TR/TE: 2500/92 ms, section thickness: $2 \mathrm{~mm}$, FOV: $6 \mathrm{~cm}$, matrix: $256 \times 256$, NEX: 1), T1WI (TR/TE: $867 / 20$ ms, section thickness: $2 \mathrm{~mm}$, FOV: $6 \mathrm{~cm}$, matrix: $256 \times 256$, NEX: 1), T2 FLAIR (TR/TE/TI: 12827/120/2250 ms, section thickness: 2 mm, FOV: $6 \mathrm{~cm}$, matrix: $352 \times 224$, NEX: 1 ), gradient-recalled echo (TR/TE/flip angle: $550 \mathrm{~ms} / 20 \mathrm{~ms} / 20^{\circ}$, section thickness: 2 mm, FOV: $6 \mathrm{~cm}$, matrix: $224 \times 224$, NEX: 2 ), and spin-echo DWI $(b=1000$, FOV: $4 \mathrm{~cm})$.

\section{Infarct Identification and Volume Measurement}

Infarct volume was measured in a randomized, blinded fashion at both 6 and 24 hours on T2WI and DWI, processed on a separate workstation with iPlan, Version 2.6 (BrainLab Software, Feldkirchen, Germany). Semiautomatic segmentation of the infarcted areas and of the entire brain was performed, and both the relative (volume of infarcted hemisphere / volume of total brain $\times 100$ ) and the absolute volume were calculated.

\section{Hemorrhage Identification and Measurement}

After the 24-hour brain MR imaging, rats were sacrificed. The brain was sliced into six 2-mm coronal sections (12 faces). The sections were fixed in $4 \%$ paraformaldehyde for 15 minutes. A previously reported visual method was used to estimate the ICH area. ${ }^{10-12}$ Both sides of the section were optically scanned. The incidence of hemorrhage was first calculated as number of rats with hemorrhage/total number of rats per group, then similar calculations were made regarding a distinct location of the hemorrhage in the basal ganglia or cortex. The hemorrhagic area on each section face was measured with the MCID imaging system (Imaging Research, St. Catherines, Ontario, Canada). ${ }^{12}$ Finally, the total area for the 12 faces was obtained by summation of individual areas.

\section{Statistical Analysis}

Statistical analysis was performed with the Statistical Package for the Social Sciences for Windows, Version 16.0 (IBM, Armonk, New York). All data were described as mean \pm SE. The difference among the 3 groups for quantitative variables of ischemia was assessed by using a 1-way ANOVA analysis. When statistical significance was obtained in the ANOVA analysis, a post hoc analysis between each of the 2 groups was assessed by using the Fisher Least Significant Difference test. The difference among the 3 groups for qualitative variables was assessed by using the $\chi^{2}$ test or Kruskal-Wallis test.

The distribution of the quantitative variable "hemorrhagic area" 
Table 1: Absolute and relative infarct volumes at 6 and 24 hours after ischemia

\begin{tabular}{|c|c|c|c|c|c|c|}
\hline \multirow[b]{2}{*}{ Group } & \multicolumn{2}{|c|}{$\begin{array}{c}\text { Relative Infarct Volume } \\
\text { (\% Total Brain) }\end{array}$} & \multicolumn{2}{|c|}{$\begin{array}{l}\text { Absolute Infarct Volume: } \\
\qquad 2 \mathrm{WI}\left(\mathrm{mm}^{3}\right)\end{array}$} & \multicolumn{2}{|c|}{$\begin{array}{c}\text { Absolute Infarct Volume: } \\
\text { DWI }\left(\mathrm{mm}^{3}\right)\end{array}$} \\
\hline & 6 hours & 24 hours & 6 hours & 24 hours & 6 hours & 24 hours \\
\hline lodixanol & $16.4 \pm 6(n=9)$ & $22.7 \pm 10(n=8)$ & $242 \pm 89(n=9)$ & $341 \pm 147(n=8)$ & $208 \pm 90(n=7)$ & $328 \pm 144(n=7)$ \\
\hline lopamidol & $22.8 \pm 5.5(n=12)$ & $32 \pm 6.3(n=10)$ & $324 \pm 70(n=12)$ & $470 \pm 91(n=10)$ & $309 \pm 49(n=10)$ & $438 \pm 90(n=9)$ \\
\hline Saline & $24.4 \pm 6.2(n=9)$ & $32.4 \pm 5.5(n=6)$ & $345 \pm 92(n=9)$ & $462 \pm 71(n=6)$ & $331 \pm 103(n=8)$ & $468 \pm 63(n=5)$ \\
\hline Statistics (ANOVA) & $P<.018$ & $P<.028$ & $P<.03$ & $P<.047$ & $P<.018$ & n.s. \\
\hline
\end{tabular}

Note:-n.s. indicates not significant.
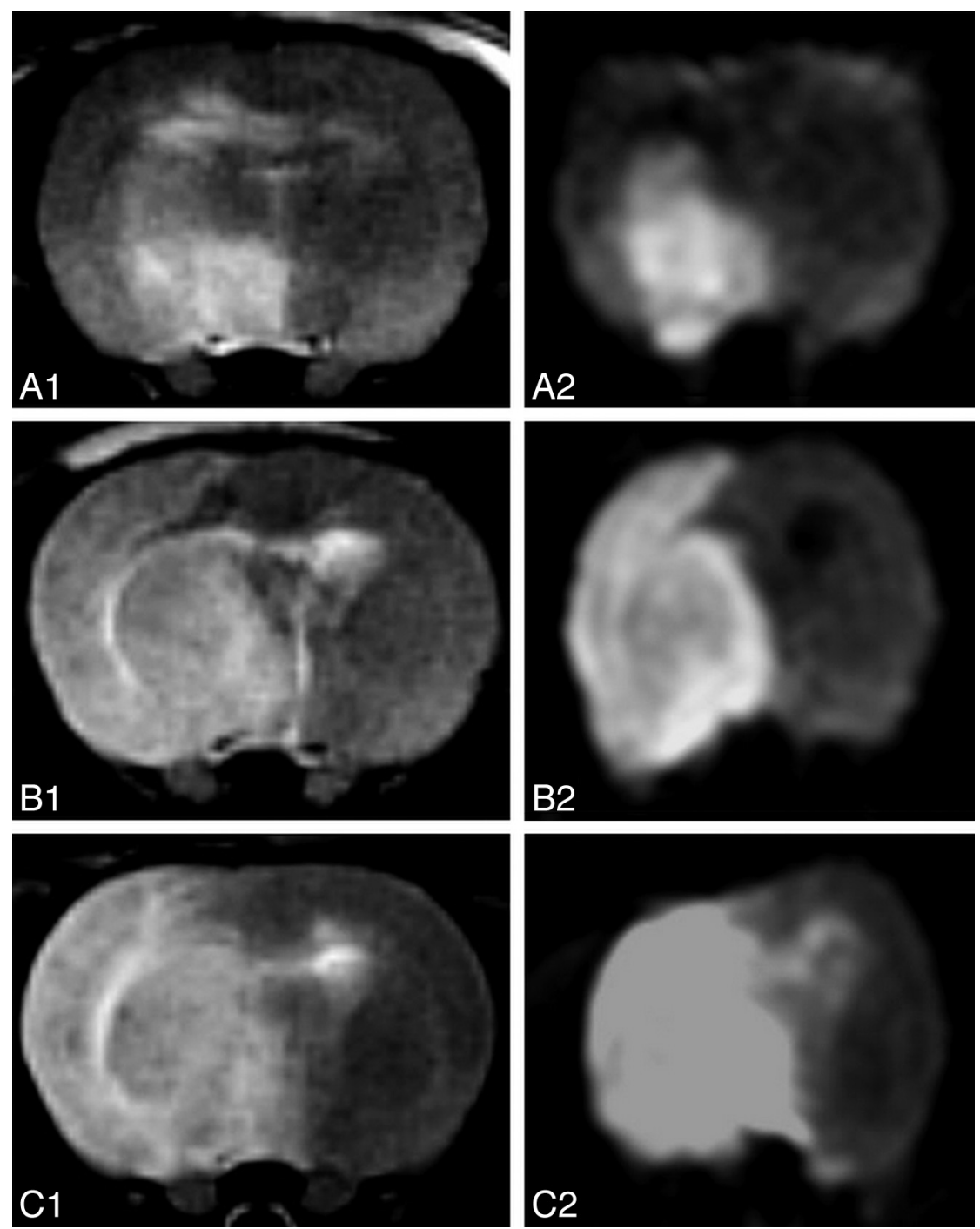

FIG 1. T2WI $(A 1, B 1, C 7)$ and DWI $(A 2, B 2, C 2)$ of 3 different rats at 24 hours after ischemia, corresponding to the iodixanol $(A 1, A 2)$, iopamidol $(B 1, B 2)$, and saline $(C 1, C 2)$ groups, show decreased infarcted volume in the iodixanol group compared with iopamidol and saline groups. showed hyperthermia, with similar average body temperatures (Celsius) in all 3 groups: $39.2 \pm 0.9$ (iodixanol), $39 \pm 0.8$ (iopamidol), and $39.3 \pm 0.9$ (saline).

$\mathrm{CBF}$ measured as the percentage perfusion after ischemia/before ischemia ( $39 \pm 26 \%, 39 \pm 17 \%$, and $46 \pm 22 \%$ ) or as the percentage perfusion after IRCM/ before ischemia $(88 \pm 14 \%, 90 \pm 18 \%$, and $78 \pm 16 \%$ ) for iodixanol, iopamidol, and saline, respectively, demonstrated no statistical difference among groups.

No statistical difference was found for the average neurologic score after $45 \mathrm{~min}$ utes and at 2, 12, and 24 hours among groups $(3.9 \pm 0.2,3.6 \pm 0.3,3.4 \pm 1$, and $3.2 \pm 1$ in the iodixanol group; $3.7 \pm 0.4$, $3.5 \pm 0.7,3.9 \pm 1.1$, and $3.7 \pm 1.3$ in the iopamidol group; $4.1 \pm 0.3,3.3 \pm 0.3,4.3 \pm$ 1.3 , and $4.5 \pm 1.8$ in the saline group, respectively) (Kruskal-Wallis test). One animal in the iodixanol group (11\%), 2 animals in the iopamidol group (17\%), and 5 animals in the saline group (55\%) died; 3, at 7 hours and 2 animals at 18 hours. Differences among groups for mortality were not statistically significant (exact test).

All animals developed T2 and FLAIR hyperintense signal and restricted diffusion in the MCA territory, including the basal ganglia, at 6 and 24 hours. ${ }^{6,13}$ Both the relative and absolute infarct volumes after administration of iodixanol were significantly smaller than those in the other 2 groups at 6 and 24 hours (Table 1 and Fig 1). Differences in infarct volumes among groups were significant at 6 hours $(P<.03)$ and 24 hours $(P<.05)($ Fig 2). Post hoc analysis for was not normal; therefore, a nonparametric (Kruskal-Wallis) test was used to compare mean areas among groups. The difference among groups for categoric variables was assessed by using the exact test. The Pearson coefficient was used to correlate infarct volume and hemorrhagic area. Statistical significance was set at $P<.05$.

\section{RESULTS}

No statistically significant differences were found among groups in body weight, morphometric parameters, or physiologic parameters as measured during surgery, including body temperature (Celsius). Following occlusion before reperfusion, all animals infarct volume differences between the iodixanol and iopamidol groups and between the iodixanol and saline groups was significant at 6 hours $(P<.03$ and $P<.01$, respectively) and 24 hours $(P<.02$ and $P<.008$, respectively).

Excessive DWI artifacts due to inadequate coil optimization were encountered in the first 4 animals at the 24-hour scan. The artifacts problem was resolved for the remainder of the study. Nevertheless, a strong trend for decreased DWI volume for the iodixanol group was identified when comparing the absolute DWI infarct volumes at 24 hours. 
All animals showed HT, typically visualized on pathologic brain sections as macroscopic non-space-occupying red areas (Fig 3). The absolute cortical ICH in the iodixanol group was significantly smaller than that in the other 2 groups $(P<.01)$ (Table 2). The incidence of cortical and deep (basal ganglia) ICH was not different among groups (exact test).

There was a positive but nonrobust linear scatterplot correlation between the MR imaging infarct volume and total hemorrhagic area on cut sections when all the groups were pooled (Pearson $\left.r=0.415, P<.023 ; r^{2}=0.17\right)$. The correlations were positive for each group and fell into the moderate category as well: saline, $r=0.365, P<.335$; iodixanol, $r=0.449, P<.225$; and iopamidol, $r=0.512, P<.089$ (Fig 4 ).

\section{DISCUSSION}

Both IV and IA IRCM injection may increase the permeability of the blood-brain barrier under normal conditions in animals. ${ }^{14-16}$ Theoretically, it is possible that large IV or IA doses of IRCM (as used in therapeutic revascularization procedures) may contribute to a BBB opening, edema, and HT. ${ }^{16,17}$ Following ischemia in

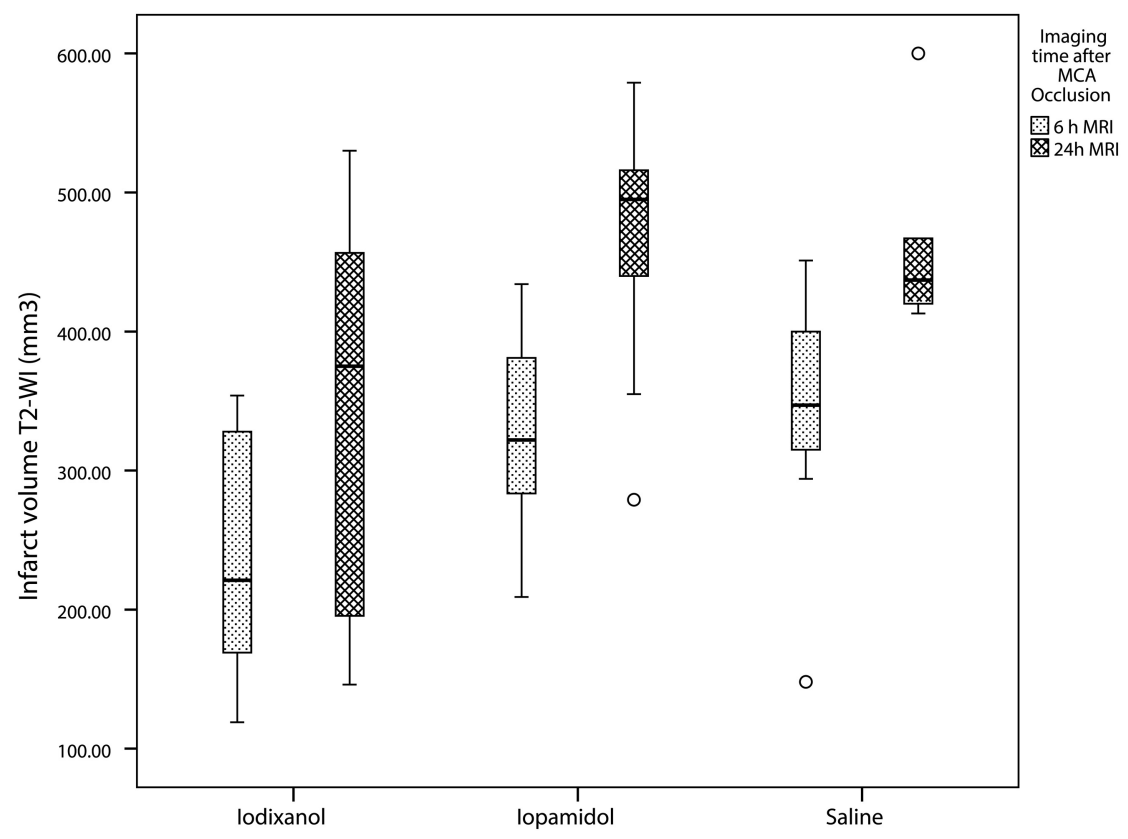

FIG 2. Boxplot analysis of absolute infarct volume per group and at 2 different times after ischemia ( 6 hours and 24 hours). There is increased volume of infarct in the iopamidol and saline groups compared with the iodixanol group.
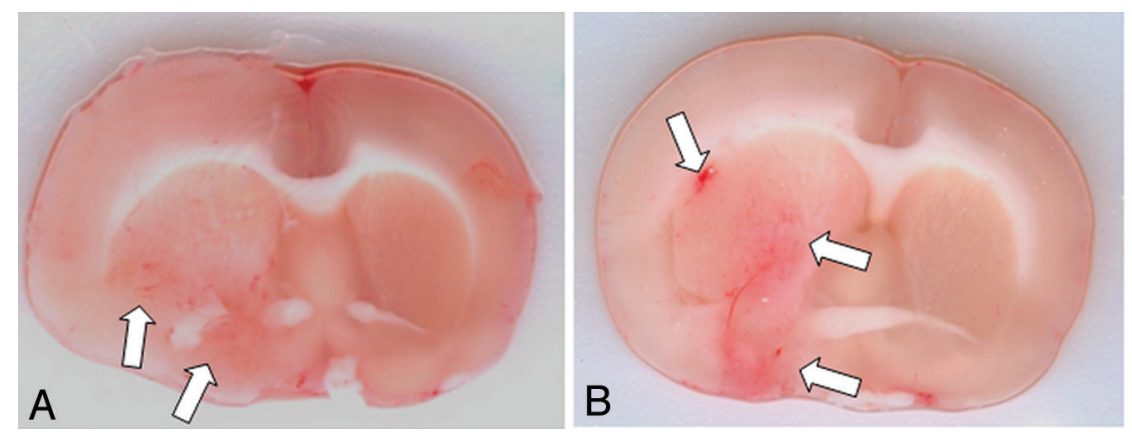

FIG 3. Hemorrhagic transformation on sample animals. $A$, lodixanol group. $B$, lopamidol group. $C$, Saline group. Note the small amount of hemorrhage in the iodixanol rat (arrows in A) compared with larger areas in the other 2 animals (arrows in $B$ and C). 
Table 2: Incidence and area of cortical and deep intracranial hemorrhage on cut-section inspection

\begin{tabular}{|c|c|c|c|c|c|c|}
\hline \multirow[b]{2}{*}{ Group } & \multicolumn{2}{|c|}{ Cortex } & \multicolumn{2}{|c|}{ Deep (Basal Ganglia) } & \multicolumn{2}{|c|}{ Total } \\
\hline & Incidence $^{b}$ & $\begin{array}{c}\text { Area } \\
\left(\mathrm{mm}^{2}\right)^{\mathrm{a}}\end{array}$ & Incidence & $\begin{array}{c}\text { Area } \\
\left(\mathrm{mm}^{2}\right)^{\mathrm{b}}\end{array}$ & Incidence & $\begin{array}{c}\text { Area } \\
\left(\mathrm{mm}^{2}\right)^{\mathrm{b}}\end{array}$ \\
\hline lodixanol $(n=9)$ & $5(56 \%)$ & $0.8 \pm 1.5$ & $9(100 \%)$ & $28 \pm 23$ & $9(100 \%)$ & $28.8 \pm 23.3$ \\
\hline lopamidol ( $n=12)$ & $9(75 \%)$ & $18.2 \pm 31$ & $12(100 \%)$ & $31 \pm 24.6$ & $12(100 \%)$ & $49.2 \pm 50.3$ \\
\hline Saline $(n=9)$ & $8(89 \%)$ & $25.7 \pm 39.7$ & $9(100 \%)$ & $56.7 \pm 29.3$ & 9 (100\%) & $82.5 \pm 62.7$ \\
\hline
\end{tabular}

a $p<.01$.

${ }^{\mathrm{b}}$ Not significant.

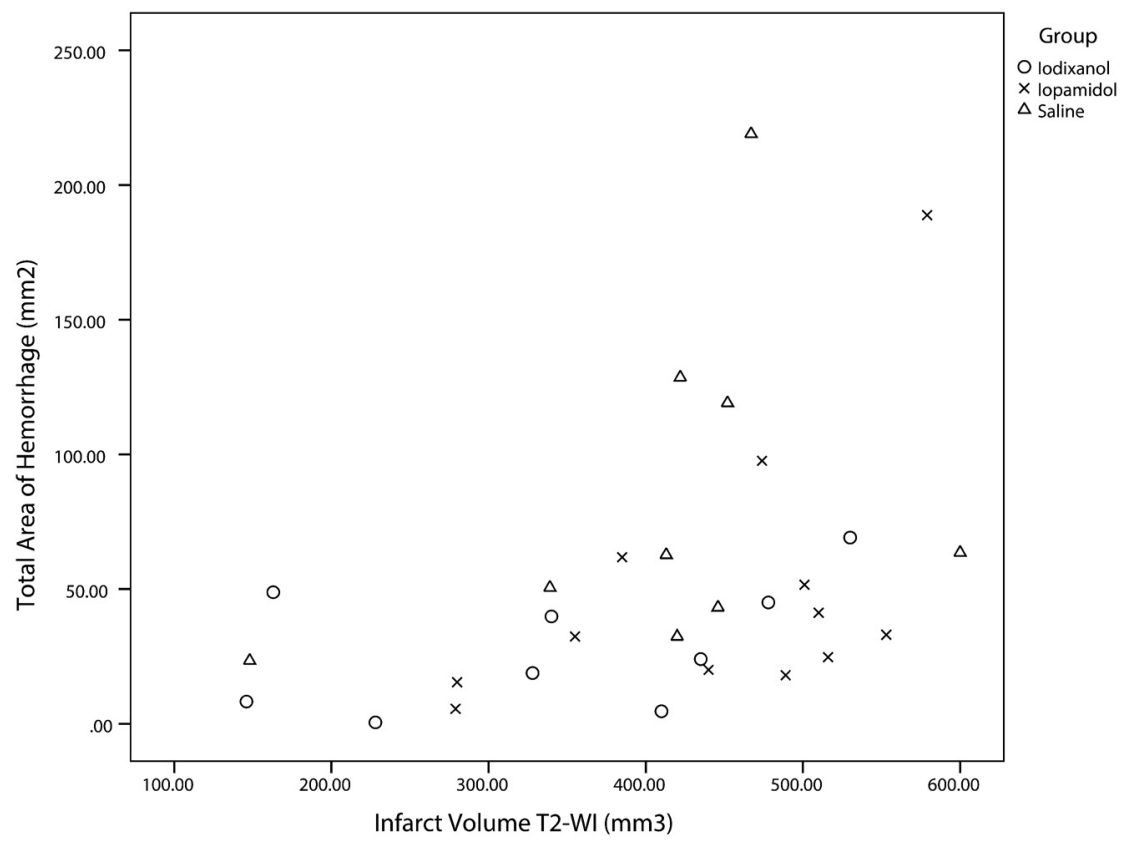

FIG 4. Scatterplot shows positive linear correlation between infarct volume and hemorrhagic transformation in all groups. The correlation is mild ( $r=0.415)$, and in some cases, large infarct volumes were not associated with large hemorrhagic areas.

able to traverse early damage to the blood-brain barrier to exert adverse osmotic or direct toxic effects in the extracellular space. Conversely, iopamidol may ultimately more easily traverse the damaged membrane to increase edema, rather than decrease it, by osmotic effect.

Endothelial cell morphology and function may be directly affected variably by IRCM injection, contributing to IRCM and fluid passage beyond the arterial lumen. ${ }^{24}$ There is conflicting evidence regarding increased neurotoxicity once the IRCM has crossed the BBB in animals and humans. ${ }^{17,25-29}$ Heinrich et $\mathrm{al}^{30}$ compared the direct cytotoxic effects of dimeric iso-osmolar and monomeric isoosmolar IRCM on renal tubular cells in vitro and found that dimeric IRCM have stronger direct cytotoxic effects, postulating a mechanism independent from osmolarity. ${ }^{30}$

Molecular chemotoxicity, related to the chemical structure of the molecule, decreases as the number of carboxyl groups decreases and the number of hydroxyl groups increases. Increasing the number of hydroxyl groups also increases the solubility of the agent, thus reducing its tendency to bind to tissues and proteins. ${ }^{31}$ Agents with no carboxyl groups and a number of hydroxyl groups evenly distributed around the main molecule have a reduced propensity for neurotoxicity. ${ }^{32}$ Iodixanol has an increased number of hydroxyl groups compared with low-osmolar IRCM.
Hydrolysis of iodixanol in vitro can produce a derivative of propylene glycol (2,3-dihydroxy-1-propylamine $\mathrm{HOCH}_{2}-\mathrm{CH}$ $\left.(\mathrm{OH})-\mathrm{CH}_{2}-\mathrm{NH}_{2}\right)$. Propylene glycol when injected intra-arterially in an ischemic brain model of rats has been found to decrease BBB dysfunction by a "sealing" effect, with subsequent decreased permeability and infarct size. $^{33}$ Although iodixanol has no biotransformation after intravenous injection, a similar "sealing" effect in the BBB is possible. ${ }^{34,35}$

Clot formation and coagulation interactions by IRCM also have impact on infarct volume and $\mathrm{ICH} .{ }^{36}$ Mechanisms and effects may be paradoxical, with equilibrium among thrombotic, antiplatelet, and fibrinolytic properties resulting in a dominant state, which varies among different IRCM. Specifically, iodixanol has been found to have a less thrombotic effect than iopamidol. ${ }^{37}$ However, no Dopplermeasured CBF differences were identified postreperfusion in our animals that could reasonably explain an effect of persistent reduced blood flow on infarct volume in the iodixanol group. Even in the absence of coagulation-mediated oligemic effects, increased hemoglobin binding attributed to IRCM may exert a circulatory hypoxic effect, promoting neurotoxicity via reduced oxygen delivery to tissue. ${ }^{38}$

Physical properties such as viscosity differ among IRCM and can exert a hydrostatic effect. ${ }^{39}$ Iodixanol has increased viscosity $(11.8 \mathrm{cP})$ compared with iopamidol $(4.7 \mathrm{cP})$ at $37^{\circ} \mathrm{C}$. The reduced hydrostatic effect across an injured $\mathrm{BBB}$ may account for the reduced infarct edema volume measured compared with saline or a less viscous low-osmolar contrast. Differences in infusion duration were not encountered in our study. Higher viscosity fluid may reduce antegrade reperfusion and reduce hydrostatic infarct edema. If so, reduced viscosity-related antegrade reperfusion may represent a new therapeutic option for further exploration.

Changes in the environment, such as decreased $\mathrm{pH}$ or increased temperature in the hypoxic brain tissue, can cause changes in the physicochemical properties of IRCM as well. The role these differences play in our particular results is difficult to postulate.

We observed a trend toward increased mortality and worse neurologic score in the saline group compared with iodixanol and iopamidol. It is likely that significant damage of the BBB had already occurred by the time of reperfusion 5 hours after ischemia. Reperfusion itself caused an inflammatory cytotoxic effect, with increased edema and ICH. Reperfusion increases levels of reactive oxygen species, including the superoxide radical and nitric oxide. ${ }^{40,41}$ Oxidative radicals trigger activation of metallopro- 
teinases, which disrupt permeability barriers and, in turn, potentiate injury to microvasculature and neural cells during ischemia and reperfusion. ${ }^{42,43}$ Larger infarcts and increased HT occur in the reperfusion of MCA occlusion compared with permanent occlusion, and metalloproteinases are particularly increased after reperfusion compared with models of permanent occlusion. ${ }^{12}$

Aquaporin-4 has been linked to the severity of infarct volume but not with HT. ${ }^{44}$ Therefore, the mechanism of HT, though linked to mechanisms affecting reperfusion, infarct volume, and edema previously discussed, might have a separate and different pathophysiologic pathway after reperfusion in acute ischemic stroke in humans and animals. ${ }^{45}$

The contributions of hemorrhagic changes, typically petechial in the rat, might also increase differences in apparent, measurable secondary infarct edema. However, on the basis of our pilot work, more hemorrhagic changes were anticipated in the IRCM group, as opposed to lesser areas with iodixanol. In contrast to our pilot work, in which $37 \%$ of saline-infused and $75 \%$ of iohexol-infused rats exhibited HT, all animals in the current study exhibited HT, most frequent and voluminous in the saline and iopamidol groups. The higher percentage of ICH in all groups can be attributed to the additional burden of postreperfusion transportation, anesthesia, MR imaging, recovery, and delay before sacrifice, likely contributing to stressinduced infarct volume and HT. Nevertheless, we identified decreased HT in the iodixanol group. Thus, we might indeed be identifying a direct or indirect effect of iodixanol, whose exact mechanisms and significance remain to be clarified.

There are a number of limitations of our methods, analyses, findings, and hypotheses regarding the IRCM effect on infarct volume and ICH. First, our data for infarct volume as measured by DWI are inconclusive at 24 hours, mainly due to field inhomogeneity artifacts precluding 4 early cases from adequate evaluation. The role of ADC for prediction of HT in our population is yet to be explored further. ${ }^{46-48}$ The 5-hour time window is later than typically used in ischemic rat models. Knight et al ${ }^{49}$ reported no T2 signal changes early after reperfusion in a model of 2-4 hours of transient ischemia. Thus, the late time was chosen to guarantee maximal ischemic potential.

The large volume and concentration of prolonged IA IRCM injection was chosen not only to avoid overlooking any potential adverse effect not obvious with lower volumes but also to more closely simulate the effect of frequent, repetitive injections in the setting of prolonged occlusion, as is commonly used in human stroke intervention, in the timeframe when intervention is most typically applied and when benefits of revascularization are expected to be diminishing.

Another limitation is the lack of images before reperfusion. Any proposed protective effect of iodixanol might have been demonstrated by different rates of increase, or reduction, of infarct edema volume between prereperfusion and postreperfusion scans. With surgery performed remote from the scanner site, imaging during occlusion was impractical at this stage of investigation.

Differences in infarct volume and ICH might be attributed to inadequate MCA occlusion or reduced reperfusion effect in the iodixanol group. However, Doppler CBF showed no statistical differences among groups, with overall no indication of inadequate occlusion or differences in reperfusion. In addition, we have found spontaneous hyperthermia useful in estimating successful occlusion of the MCA territory in our model, with direct correlation between the body temperature and infarct size. ${ }^{50}$ All animals showed hyperthermia with similar average body temperatures before reperfusion in all 3 groups. Therein, we have no evidence that the infarct development before reperfusion was different in all 3 groups.

Nevertheless, further investigation with a larger number of animals could improve the consistency of late DWI measurements; if possible, MR imaging before reperfusion could help to confirm our results.

\section{CONCLUSIONS}

In our MCA occlusion/reperfusion rat model, iso-osmolar iodixanol perfusion demonstrated reduced infarct volume and cortical ICH area compared with both low-osmolar iopamidol and saline perfusion. The responsible mechanism for these findings is not clear. Multiple mechanisms are certainly possible, including not only physical-chemical or coagulation-related effects but also antioxidative or other antitoxic effects at the BBB and/or in the brain parenchyma. Although different low-osmolar IRCM can have distinct effects in the infarcted brain, a potential direct or indirect effect of iodixanol might be postulated. Further investigation is warranted.

\section{ACKNOWLEDGMENTS}

The authors thank engineers Enrique Neira and Carlos Morales (Department of Chemistry, Universidad Nacional de Ingenieria, Lima-Peru) for their valuable contribution to our understanding of the chemical properties of the contrast agents discussed in this article.

Disclosures: Humberto Morales—RELATED: Grant: Foundation of the American Society of Neuroradiology, ${ }^{*}$ Comments: Financial support by budget was distributed for supplies, scans, and surgical assistance. Aigang Lu—RELATED: Grant: American Society of Neuroradiology. * Thomas Tomsick—RELATED: Grant: Foundation of the American Society of Neuroradiology, ${ }^{\star}$ Society for Interventional Radiology, ${ }^{*} \mathrm{Com}$ ments: Grant funds were not used for my salary support, but rather for supplies, scans, and surgical assistance, Provision of Writing Assistance, Medicines, Equipment, or Administrative Support: Foundation of the American Society of Neuroradiology, ${ }^{*}$ Society for Interventional Radiology, ${ }^{\star}$ Comments: Grant funds were used for equipment and surgical support. ${ }^{\star}$ Money paid to the institution.

\section{REFERENCES}

1. Hom J, Dankbaar JW, Soares BP, et al. Blood-brain barrier permeability assessed by perfusion CT predicts symptomatic hemorrhagic transformation and malignant edema in acute ischemic stroke. AJNR Am J Neuroradiol 2011;32:41-48

2. Aviv RI, d'Esterre CD, Murphy BD, et al. Hemorrhagic transformation of ischemic stroke: prediction with CT perfusion. Radiology 2009;250:867-77

3. Lin K, Kazmi KS, Law M, et al. Measuring elevated microvascular permeability and predicting hemorrhagic transformation in acute ischemic stroke using first-pass dynamic perfusion CT imaging. AJNR Am J Neuroradiol 2007;28:1292-98

4. Lummel N, Schulte-Altedorneburg G, Bernau C, et al. hyperattenuated intracerebral lesions after mechanical recanalization in acute stroke. AJNR Am J Neuroradiol 2014;35:345-51

5. Kurosawa Y, Lu A, Khatri P, et al. Intra-arterial iodinated radiographic contrast material injection administration in a rat middle cerebral artery occlusion and reperfusion model: possible effects on intracerebral hemorrhage. Stroke 2010;41:1013-17

6. Koizumi A, Yoshida Y, Nakazawa T, et al. Experimental studies of ischemic brain edema: a new experimental model of cerebral em- 
bolism in rats in which recirculation can be introduced in the ischemic area. Jpn J Stroke 1986;8:1-8

7. Longa EZ, Weinstein PR, Carlson S, et al. Reversible middle cerebral artery occlusion without craniectomy in rats. Stroke 1989;20:84-91

8. Nestler U, Seifner S, Greschus S, et al. Doppler ultrasonographic measurement of blood flow velocities in major cerebral arteries of the rat using triplex mode. Neurol Res 2006;28:877-80

9. Zhang RL, Chopp M, Zhang ZG, et al. A rat model of focal embolic cerebral ischemia. Brain Res 1997;766:83-92

10. Lapchak PA, Chapman DF, Zivin JA. Metalloproteinase inhibition reduces thrombolytic (tissue plasminogen activator)-induced hemorrhage after thromboembolic stroke. Stroke 2000;31:303440

11. Lapchak PA, Araujo DM, Song D, et al. The nonpeptide glycoprotein IIb/IIIa platelet receptor antagonist SM-20302 reduces tissue plasminogen activator-induced intracerebral hemorrhage after thromboembolic stroke. Stroke 2002;33:147-52

12. Lu A, Clark JF, Broderick JP, et al. Mechanical reperfusion is associated with post-ischemic hemorrhage in rat brain. Exp Neurol 2009;216:407-12

13. Edvinsson L, MacKenzie ET, McCulloch J. General and comparative anatomy of the cerebral circulation. In: Edvinsson L, MacKenzie ET, McCulloch J. Cerebral Blood Flow and Metabolism. New York: Raven Press; 1993:3-39

14. Aulie Michelet A. Effects of intravascular contrast media on bloodbrain barrier: comparison between iothalamate, iohexol, iopentol and iodixanol. Acta Radiol 1987;28:329-33

15. Sage MR, Wilson AJ, Scroop R. Contrast media and the brain: the basis of CT and MR imaging enhancement. Neuroimaging Clin NAm 1998;8:695-707

16. Sage MR, Wilcox J, Evill CA, et al. Comparison of blood-brain barrier disruption by intracarotid iopamidol and methylglucamine iothalamate. AJNR Am J Neuroradiol 1983;4:893-95

17. Hayakawa K, Morris TM, Katzberg RW. Opening of the blood-brain barrier by intravenous contrast media in euvolemic and dehydrated rabbits. Acta Radiol 1989;30:439-44

18. Doerfler A, Engelhorn T, von Kummer R, et al. Are iodinated contrast agents detrimental in acute cerebral ischemia? An experimental study in rats. Radiology 1998;206:211-17

19. Pannu N, Wiebe N, Tonelli M. Prophylaxis strategies for contrastinduced nephropathy. JAMA 2006;295:2765-79

20. Fountaine H, Harnish $\mathrm{P}$, Andrew E, et al. Safety, tolerance, and pharmacokinetics of iodixanol injection, a nonionic, isosmolar, hexaiodinated contrast agent. Acad Radiol 1996(3 suppl 3):S475-84

21. Finley Caulfield A, Wijman CA. Management of acute ischemic stroke. Neurol Clin 2008;26:345-71, vii

22. Diringer MN, Zazulia AR. Osmotic therapy: fact and fiction. Neurocrit Care 2004;1:219-33

23. Qureshi AI, Wilson DA, Traystman RJ. Treatment of elevated intracranial pressure in experimental intracerebral hemorrhage: comparison between mannitol and hypertonic saline. Neurosurgery 1999;44:1055-63, discussion 1063-64

24. Franke RP, Fuhrmann R, Hiebl B, et al. Influence of various radiographic contrast media on the buckling of endothelial cells. Microvasc Res 2008;76:110-13

25. Wilcox J, Wilson AJ, Evill CA, et al. A comparison of blood-brain barrier disruption by intracarotid iohexol and iodixanol in the rabbit. AJNR Am J Neuroradiol 1987;8:769-72

26. Macdougall NJ, McVerry F, Baird S, et al. Iodinated contrast media and cerebral hemorrhage after intravenous thrombolysis. Stroke 2011;42:2170-74

27. Khatri P, Broderick JP, Khoury JC, et al. Microcatheter contrast injections during intra-arterial thrombolysis may increase intracranial hemorrhage risk. Stroke 2008;39:3283-87

28. Leong S, Fanning NF. Persistent neurological deficit from iodinated contrast encephalopathy following intracranial aneurysm coiling: a case report and review of the literature. Interv Neuroradiol 2012;18:33-41
29. Hayman LA, Evans RA, Bastion FO, et al. Delayed high dose contrast CT: identifying patients at risk of massive hemorrhagic infarction. AJR Am J Roentgenol 1981;136:1151-59

30. Heinrich MC, Kuhlmann MK, Grgic A, et al. Cytotoxic effects of ionic high-osmolar, nonionic monomeric, and nonionic iso-osmolar dimeric iodinated contrast media on renal tubular cells in vitro. Radiology 2005;235:843-49

31. McClennan BL, Stolberg HO. Intravascular contrast media: ionic versus nonionic-current status. Radiol Clin North Am 1991; 29:437-54

32. Dooley M, Jarvis B. Iomeprol: a review of its use as a contrast medium. Drugs 2000;59:1169-86

33. Sood R, Taheri S, Estrada EY, et al. Quantitative evaluation of the effect of propylene glycol on BBB permeability. J Magn Reson Imaging 2007;25:39-47

34. Jacobsen PB, Larsen A, Konarboland R, et al. Biotransformation of nonionic $\mathrm{x}$-ray contrast agents in vivo and in vitro. Drug Metab Dispos 1999;27:1205-13

35. Spencer CM, Goa KL. Iodixanol: a review of its pharmacodynamic and pharmacokinetic properties and diagnostic use as an $\mathrm{x}$-ray contrast medium. Drugs 1996;52:899-927

36. Aspelin P, Stacul F, Thomsen HS, et al. Effects of iodinated contrast media on blood and endothelium. Eur Radiol 2006;16:1041-49

37. Georgakis A, Ener RA, Jin J, et al. Risk of thrombogenicity among nonionic radiocontrast agents. I Invasive Cardiol 2008;20: 349-53

38. Kim SJ, Salem MR, Joseph NJ, et al. Contrast media adversely affect oxyhemoglobin dissociation. Anesth Analg 1990;71:73-76

39. Wilcox J, Sage MR. Is viscosity important in the production of blood-brain barrier disruption by intracarotid contrast media? Neuroradiology 1984;26:511-13

40. Dirnagl U, Lindauer U, Them A, et al. Global cerebral ischemia in the rat: online monitoring of oxygen free radical production using chemiluminescence in vivo. J Cereb Blood Flow Metab 1995; 15:929-40

41. Kumura E, Yoshimine T, Iwatsuki KI, et al. Generation of nitric oxide and superoxide during reperfusion after focal cerebral ischemia in rats. Am J Physiol 1996;270:C748-52

42. Rosell A, Foerch C, Murata Y, et al. Mechanisms and markers for hemorrhagic transformation after stroke. Acta Neurochir Suppl 2008; 105:173-78

43. Lu A, Clark JF, Broderick JP, et al. Reperfusion activates metalloproteinases that contribute to neurovascular injury. Exp Neurol 2008;210:549-59

44. Zador Z, Stiver S, Wang V, et al. Role of aquaporin-4 in cerebral edema and stroke. Handb Exp Pharmacol 2009:159-70

45. Simard JM, Kent TA, Chen M, et al. Brain oedema in focal ischaemia: molecular pathophysiology and theoretical implications. Lancet Neurol 2007;6:258-68

46. Kim EY, Na DG, Kim SS, et al. Prediction of hemorrhagic transformation in acute ischemic stroke: role of diffusion-weighted imaging and early parenchymal enhancement. AJNR Am J Neuroradiol 2005;26:1050-55

47. Tong DC, Adami A, Moseley ME, et al. Prediction of hemorrhagic transformation following acute stroke: role of diffusion- and perfusion-weighted magnetic resonance imaging. Arch Neurol 2001;58:587-93

48. Hoehn-Berlage M, Norris DG, Kohno K, et al. Evolution of regional changes in apparent diffusion coefficient during focal ischemia of rat brain: the relationship of quantitative diffusion NMR imaging to reduction in cerebral blood flow and metabolic disturbances. J Cereb Blood Flow Metab 1995;15:1002-11

49. Knight RA, Barker PB, Fagan SC, et al. Prediction of impending hemorrhagic transformation in ischemic stroke using magnetic resonance imaging in rats. Stroke 1998;29:144-51

50. Reglodi D, Somogyvari-Vigh A, Maderdrut JL, et al. Postischemic spontaneous hyperthermia and its effects in middle cerebral artery occlusion in the rat. Exp Neurol 2000;163:399-407 